\title{
无催化剂条件下合成喹啉并吡咯并喹啉衍生物
}

\author{
赵 群 $a$ 姚昌盛 $b$ 王香善 $*, b$ \\ ( ${ }^{a}$ 南京中医药大学药学院 南京 210023) \\ ( ${ }^{b}$ 江苏师范大学化学化工学院＼cjkstart徐州 221116)
}

\begin{abstract}
摘要 以芳醛、5-氨基吲哚和 4-羟基喹啉-2-酮为原料, 在回流的乙醇中, 无需加入任何催化剂, 可高产率得到一系列 13-芳基-6,13-二氢化-3H-喹啉并[4,3-b]吡咯并[3,2-f]喹啉-12(11H)-酮衍生物，并通过 X 射线单晶衍射分析确证了 4b 的 结构. 该方法简单, 容易操作, 为稠合五环含双喹啉杂环化合物提供了有效的合成方法.

关键词＼cjkstart喹啉并吡咯并喹啉; 5-氨基吲哚; 无催化剂; 合成
\end{abstract}

\section{Synthesis of Quinopyrroloquinoline Derivatives under Catalyst-Free Conditions}

\author{
Zhao, Qun ${ }^{a} \quad$ Yao, Changsheng ${ }^{b} \quad$ Wang, Xiangshan ${ }^{*}, b$ \\ $\left({ }^{a}\right.$ College of Pharmacy, Nanjing University of Chinese Medicine, Nanjing 210023) \\ ( ${ }^{b}$ Jiangsu Key Laboratory of Green Synthesis for Functional Materials, School of Chemistry and Chemical Engineering, \\ Jiangsu Normal University, Xuzhou 221116)
}

\begin{abstract}
In this paper, a series of 13-aryl-6,13-dihydro-3H-quino[4,3-b]pyrrolo[3,2-f]quinolin-12(11H)-one derivatives was synthesized in EtOH under catalyst-free conditions using aromatic aldehydes, 5-aminoindole and 4-hydroxyquinolin-2(1H)one as reactants. The structure of $\mathbf{4 b}$ was confirmed by X-ray diffraction analysis. This procedure has the advantages of simplicity and easy operation. An efficient method for the synthesis of fused pentacyclic heterocycles containing biquinolines moieties is provided.
\end{abstract}

Keywords quinopyrroloquinoline; 5-aminoindole; catalyst-free; synthesis

众所周知, 喹啉是最具有药用价值的活性分子之 一. 其中, 值得一提的是其抗症活性 ${ }^{[1]}$. 目前很多临床

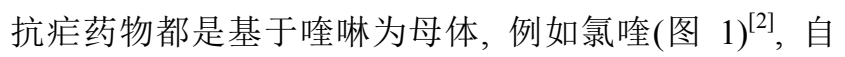
1944 年开始就被应用于临床治疗疮疾. 现在对于风湿 关节炎的治疗, 也有一定效果. 再如奎宁(图 1) ${ }^{[3]}$, 是一 种天然的生物碱, 最初分离于茜草科植物金鸡纳树及其 同属植物的树皮中. 该药物能与症原虫的 DNA 结合, 形成复合物, 抑制其 DNA 的复制和 RNA 的转录, 广泛 用于治疗症疾. 所以, 喹啉的构筑一直是有机合成化学 的研究热点 ${ }^{[4]}$. 最近采用多组分反应或多米诺反应, 逐 渐被认为是构筑喹啉骨架的最有效方法之一 ${ }^{[5]}$, 特别是 利用活性的芳胺, 与苯甲醛、活性亚甲基化合物的三组 分反应, 可以在无催化剂条件下进行, 并高产率获得结
构多样化的喹啉衍生物 ${ }^{[6]}$. 该方法首先是利用芳醛与活 性亚甲基化合物发生 Knoevenagel 缩合反应, 形成的 $\alpha, \beta$-不饱和酮再与活性的芳胺(作为烯胺)发生 Michael 加成反应; 最后分子内的氨基与羰基缩合形成喹啉骨 架. 我们实验室在这个反应方面做了很多工作, 成功构 筑了吡唑并喹啉、芸啶、吲哚并听啶等含氮杂环骨架 [7]. 最近, 我们采用 5-氨基吲哚作为活性的芳胺、4-羟基喹 啉-2-酮作为活性亚甲基化合物，与芳酫在回流的乙醇 中反应, 可以成功构筑双喹啉骨架外, 结构中还同时具 有吡咯的杂环. 本文报道系列 13-芳基-6,13-二氢化-3H喹啉并 $[4,3-b]$ 吡咯并 $[3,2-f]$ 喹啉-12(11H)-酮衍生物的合 成和产物 $4 \mathrm{~b}$ 的晶体结构.

* E-mail: xswang@jsnu.edu.cn

Received February 1, 2016; revised March 25, 2016; published online April 15, 2016.

Project supported by the Major Natural Science Foundation of Jiangsu Province (No. 14KJA150004), and a project funded by the Priority Academic Program Development of Jiangsu Higher Education Institutions.

江苏省高校重大基础研究基金(No. 14KJA150004)和江苏省高校优势学科建设工程资助项目. 
<smiles>CCN(CC)CCCC(C)Nc1ccnc2cc(Cl)ccc12</smiles>

氯喹<smiles>C=CC1=C(C=C)C[C@@H]([C@H](O)c2ccnc3ccc(OC)cc23)N=C1</smiles>

奎宁

图 1 氯喹和奎宁的结构

Figure 1 Structures of chloroquine and quinine

\section{1 结果与讨论}

将等量的芳醛(1)、5-氨基吲哚(2)和 4-羟基喹啉-2酮(3)置于无水乙醇中, 回流反应, 无需加入任何催化 剂, 即可较高产率地得到 13-芳基-6,13-二氢化-3H-喹啉 并[4,3-b]吡咯并[3,2-f]喹啉-12(11H)-酮衍生物(4) (Eq. 1).

$\mathrm{ArCHO}+$<smiles>[NH3+]c1ccc2[nH]ccc2c1</smiles>

2<smiles>N=c1c2c(=O)[nH]c3ccccc3c2[nH]c2ccc3[nH]ccc3c12</smiles>

最初根据 Knoevenagel 缩合反应和 Michael 反应发 生的条件, 我们认为碱对这个反应能起到催化或促进作 用, 所以向反应体系中加入少量的碱, 例如三乙胺、六 氢吡啶或无机碱碳酸钠等, 发现反应的产率并没有得到 提高. 反应的温度对产率对影响很大, 乙醇的回流温度 得到的产率最高 $(\mathbf{4 a}, 88 \%)$, 而且该反应的最大优点是反 应的产物无需重结晶. 反应通过 TLC 检测到 5-氨基吲 哚反应完全后, 冷却析出大量固体, 过滤即可得到纯净 的产物 4 .

同样在无催化的条件改变不同取代的苯甲醛, 例如 无论是吸电子基的卤素，还是供电子的甲基、甲氧基; 无论取代基在邻位、间位还是对位; 无论是单取代, 还 是多取代的苯甲醛, 都给出很好的收率(表 1). 化合物的 结构都通过氢谱、红外和高分辨质谱加以表征. 为了进 一步确证产物的结构, 我们以 DMF 为溶剂, 培养和测 定了产物 $4 \mathrm{~b}$ 的单晶. 产物 $4 \mathrm{~b}$ 的结构图见图 2.

$\mathrm{X}$ 光衍射分析表明新形成的吡啶环 [C (4)$\mathrm{C}(6) / \mathrm{C}(14) / \mathrm{N}(3) / \mathrm{C}(15)]$ 采用船式构象: 其中原子 $\mathrm{C}(4)$, $\mathrm{C}(6), \mathrm{C}(14)$ 和 $\mathrm{C}(15)$ 共平面 [平面 I, 平面上原子的平均偏 差为 0.009(1) $\AA$ ], 原子 $\mathrm{C}(5)$ 和 $\mathrm{N}(3)$ 偏离该平面的距离分 别为 $0.249(3)$ 和 $0.122(3) \AA$. 原先的吲哚环[平面 II,
表 1 产物 4 的反应时间和产率

Table 1 The reaction time and yields of $\mathbf{4}^{a}$

\begin{tabular}{clccc}
\hline 序号 & \multicolumn{1}{c}{$\mathrm{Ar}$} & 反应时间 $/ \mathrm{h}$ & 产物 & 产率/\% \\
\hline 1 & $4-\mathrm{BrC}_{6} \mathrm{H}_{4}$ & 10 & $\mathbf{4 a}$ & 88 \\
2 & $3-\mathrm{ClC}_{6} \mathrm{H}_{4}$ & 8 & $\mathbf{4 b}$ & 91 \\
3 & $4-\mathrm{ClC}_{6} \mathrm{H}_{4}$ & 10 & $\mathbf{4 c}$ & 84 \\
4 & $3,5-(\mathrm{MeO})_{2} \mathrm{C}_{6} \mathrm{H}_{3}$ & 6 & $\mathbf{4 d}$ & 87 \\
5 & $3,4-\left(\mathrm{CH}_{3}\right)_{2} \mathrm{C}_{6} \mathrm{H}_{3}$ & 10 & $\mathbf{4 e}$ & 85 \\
6 & $2-\mathrm{MeOC}_{6} \mathrm{H}_{4}$ & 10 & $\mathbf{4 f}$ & 90 \\
7 & $3-\mathrm{MeOC}_{6} \mathrm{H}_{4}$ & 8 & $\mathbf{4 g}$ & 87 \\
8 & $4-\mathrm{MeC}_{6} \mathrm{H}_{4}$ & 9 & $\mathbf{4 h}$ & 82 \\
9 & $2,4,5-(\mathrm{MeO})_{3} \mathrm{C}_{6} \mathrm{H}_{2}$ & 12 & $\mathbf{4 i}$ & 90 \\
\hline$a$
\end{tabular}

酮 $(161 \mathrm{mg}, 1.0 \mathrm{mmol})$, 无水乙醇 $(10 \mathrm{~mL})$, 回流.

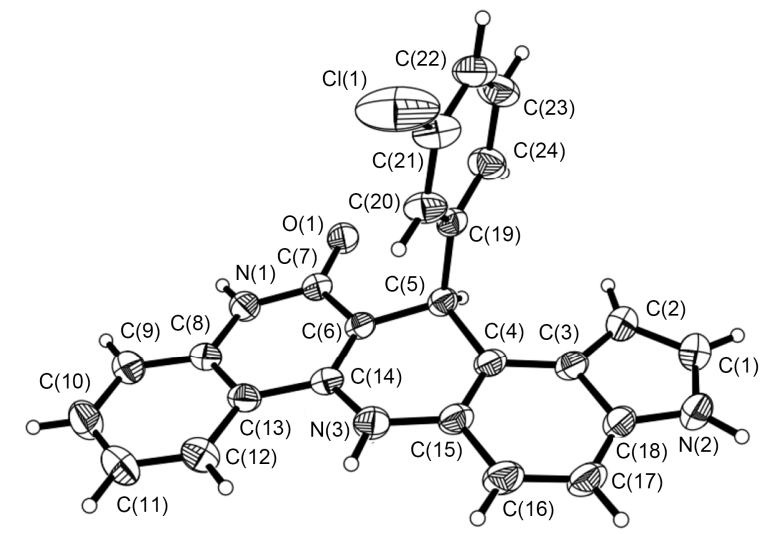

图 2 产物 $4 \mathrm{~b}$ 的晶体结构图

Figure 2 The crystal structure of product $4 \mathbf{b}$

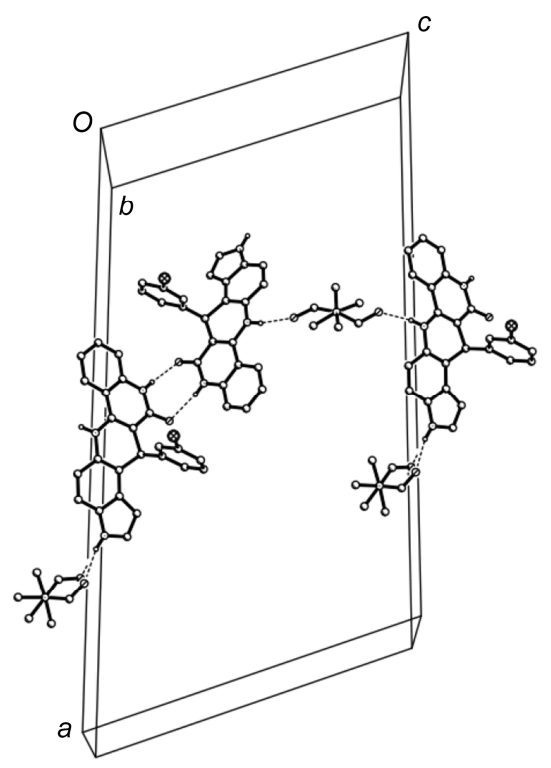

图 $34 \mathrm{~b}$ 中分子间氢键图

Figure 3 The intermolecular hydrogen bonds in $\mathbf{4 b}$

$\mathrm{C}(1)-\mathrm{C}(4) / \mathrm{C}(15)-\mathrm{C}(18) / \mathrm{N}(2)$ ]、喹啉环 [平面 III, $\mathrm{C}(6)-\mathrm{C}(14) / \mathrm{N}(1)$ ] 和苯环 [平面 IV, $\mathrm{C}(19)-\mathrm{C}(24)$ ] 均为平 面结构. 新形成的吡啶环(平面 I) 几乎平行于吲哚环(平 
面 II)和喹啉环(平面 III), 形成的二面角分别为 9.0(1)和 $6.1(1)^{\circ}$. 该平面还几乎垂直于苯环(平面 IV), 形成的二 面角为 $82.1(1)^{\circ}$.

分子中存在三个 $\mathrm{N}-\mathrm{H}$ 键, 都分别参与形成了氢键 (表 2). 其中, 喹啉环中酰胺键形成了分子间氢键 $N(1)$ $-\mathrm{H}(1) \cdots \mathrm{O}(1)$, 连接相邻的分子形成二聚体(图 3). 这个 相邻的二聚体不能自行连接, 而是通过一分子的溶剂 DMF, 首尾倒置形成 $\mathrm{N}(3)-\mathrm{H}(3) \cdots \mathrm{O}(4)$ 分子间氢键, 像 桥一样连接相邻二聚体, 沿着 $c$ 轴拓展形成多聚体. 另 外分子的吲哚环上的 $\mathrm{N}(2)-\mathrm{H}(2)$, 通过 $\mathrm{N}(2)-\mathrm{H}(2) \cdots$ $\mathrm{O}(3)$ 氢键连接另外一个溶剂 DMF 分子.

根据产物的结构, 我们认为是醛 1 先与 2-羟基喹 啉-2-酮(3)发生 Knoevenagel 缩合反应形成 $\alpha, \beta$-不饱和酮 5 ; 5 再与 5-氨基吲哚(2, 作为烯胺)发生 Michael 加成得 到中间体 6; 接着 6 分子内的氨基和羰基进行亲核加成 得到 7; 7 最终脱去一分子水形成目标产物 4. 具体过程 见 Scheme 1 .

\section{2 结论}

本文在回流的乙醇中, 无需加入任何额外的催化 剂, 可以通过醛、5-氨基吲哚和 4-差基喹啉-2-酮的三组 分反应, 一步构建稠合五环的喹啉并吡咯并喹啉衍生 物. X-光衍射分析表明新形成的 1,4-二氢吡啶环采用经 典的船式构象, 并详细讨论了平面之间的夹角和分子与 溶剂之间的氢键连接关系.

\section{3 实验部分}

\section{1 仪器与试剂}

熔点测定使用 XT-5 型数字熔点仪; 红外光谱采用 TENSOR 27 型红外光谱仪测定( $\mathrm{KBr}$ 压片); 核磁共振氢 谱采用 Bruker AVANCE DPX-400 型核磁共振仪测定, $\mathrm{DMSO}-d_{6}$ 为溶剂, TMS 为内标; 高分辨质谱在 Brukermicro-TOF-Q-MS 质谱仪上测定.

\section{2 产物 4 的合成}

干燥的 $50 \mathrm{~mL}$ 烧瓶中加入芳醛 $1(1.0 \mathrm{mmol}), 5$-氨基 吲哚(2) (132 mg, $1.0 \mathrm{mmol}$ ), 4-差基喹啉-2-酮(3) (161 $\mathrm{mg}, 1.0 \mathrm{mmol})$ 和无水乙醇 $(10 \mathrm{~mL})$. 搅拌加热回流反应 保持 6 12 h, 通过 TLC 监测反应的进程. 反应结束后, 冷却到室温, 析出大量固体, 通过简单的过滤即可得到 纯净的化合物 4 .

13-(4-溴苯基)-6,13-二氢化-3H-喹啉并 $[4,3-b]$ 吡咯 并[3,2-f]喹啉-12(11H)-酮(4a): m.p. $>300{ }^{\circ} \mathrm{C} ;{ }^{1} \mathrm{H}$ NMR (DMSO- $\left.d_{6}, 400 \mathrm{MHz}\right) \delta: 5.65(\mathrm{~s}, 1 \mathrm{H}, \mathrm{CH}), 6.40(\mathrm{~s}, 1 \mathrm{H}$, ArH), $7.20 \sim 7.31(\mathrm{~m}, 9 \mathrm{H}, \operatorname{ArH}), 7.46(\mathrm{t}, J=7.6 \mathrm{~Hz}, 1 \mathrm{H}$, $\operatorname{ArH}), 8.28$ (d, J=8.0 Hz, 1H, ArH), 9.22 (s, 1H, NH), 11.03 (s, 1H, NH), 11.12 (s, 1H, NH); IR (KBr) v: 3311, 3164, 3093, 3044, 3959, 2929, 2868, 1672, 1638, 1602, $1485,1459,1407,1371,1349,1099,1072,1013,866,839$,

表 2 晶体结构 $4 \mathrm{~b}$ 中的氢键数据

Table 2 The hydrogen bond data in the crystal structure of $\mathbf{4 b}$

\begin{tabular}{|c|c|c|c|c|c|c|}
\hline 序号 & $\mathrm{D}-\mathrm{H} \cdots \mathrm{A} / \AA$ & $d(\mathrm{D}-\mathrm{H}) / \AA$ & $d(\mathrm{H} \cdots \mathrm{A}) / \AA$ & $\angle \mathrm{DHA} /\left(^{\circ}\right)$ & $d(\mathrm{D} \cdots \mathrm{A}) / \AA$ & 对称操作 \\
\hline 1 & $\mathrm{~N}(3)-\mathrm{H}(3) \cdots \mathrm{O}(4)$ & 0.852 & 2.085 & 168.41 & 2.924 & $X-1, y, z-1$ \\
\hline 2 & $\mathrm{~N}(2)-\mathrm{H}(2) \cdots \mathrm{O}\left(3^{\prime}\right)$ & 0.881 & 1.759 & 174.52 & 2.638 & $x, y, z-1$ \\
\hline 3 & $\mathrm{~N}(2)-\mathrm{H}(2) \cdots \mathrm{O}(3)$ & 0.881 & 1.997 & 166.82 & 2.862 & $x, y, z-1$ \\
\hline 4 & $\mathrm{~N}(1)-\mathrm{H}(1) \cdots \mathrm{O}(1)$ & 0.796 & 2.058 & 170.90 & 2.847 & $-x, y,-z+1 / 2$ \\
\hline
\end{tabular}

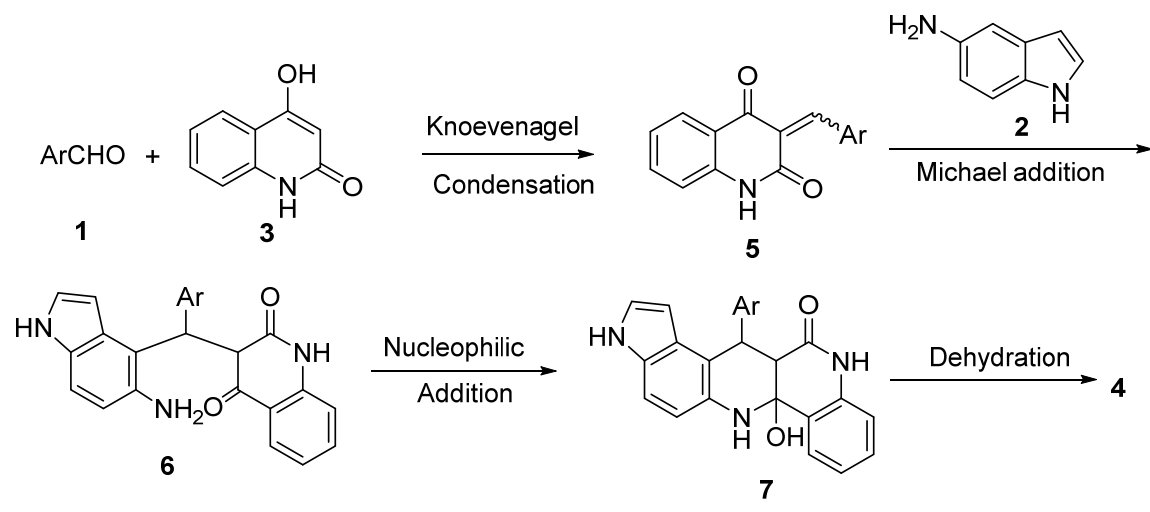

图式 1 Synthesis of compound 4

Scheme 1 化合物 $\mathbf{4}$ 的合成 
805, 750, $694 \mathrm{~cm}^{-1}$. HRMS (ESI) calcd for $\mathrm{C}_{24} \mathrm{H}_{15} \mathrm{~N}_{3} \mathrm{OBr}$ $[\mathrm{M}-\mathrm{H}]^{-}$440.0398, found 440.0415 .

13-(3-氯苯基)-6,13-二氢化-3H-喹啉并 [4,3- $b$ 吡咯 并[3,2-f]喹啉-12(11H)-酮(4b): m.p. $>300{ }^{\circ} \mathrm{C} ;{ }^{1} \mathrm{H} \mathrm{NMR}$ $\left(\mathrm{DMSO}-d_{6}, 400 \mathrm{MHz}\right) \delta: 5.72(\mathrm{~s}, 1 \mathrm{H}, \mathrm{CH}), 6.46(\mathrm{~s}, 1 \mathrm{H}$, ArH), 7.08 (d, J=8.0 Hz, 1H, ArH), $7.15 \sim 7.18(\mathrm{~m}, 1 \mathrm{H}$, ArH), $7.24 \sim 7.33(\mathrm{~m}, 7 \mathrm{H}, \mathrm{ArH}), 7.47 \sim 7.51(\mathrm{~m}, 1 \mathrm{H}, \mathrm{ArH})$, $8.30(\mathrm{~d}, J=8.0 \mathrm{~Hz}, 1 \mathrm{H}, \operatorname{ArH}), 9.28$ (s, 1H, NH), 11.07 (s, $1 \mathrm{H}, \mathrm{NH}), 11.14$ (s, 1H, NH); IR (KBr) v: 3276, 3169, 3096, 3051, 2954, 2930, 2875, 2824, 1667, 1636, 1594, 1564, 1487, 1460, 1409, 1390, 1351, 1242, 1101, 888, 777, $748,692 \mathrm{~cm}^{-1}$. HRMS (ESI) calcd for $\mathrm{C}_{24} \mathrm{H}_{17} \mathrm{~N}_{3} \mathrm{OCl}[\mathrm{M}+$ $\mathrm{H}]^{+}$396.0904, found 396.0905.

13-(4-氯苯基)-6,13-二氢化-3H-喹啉并[4,3- $b$ 吡咯 并[3,2-f]喹啉-12(11H)-酮(4c): m.p.>300 ${ }^{\circ} \mathrm{C} ;{ }^{1} \mathrm{H}$ NMR $\left(\mathrm{DMSO}-d_{6}, 400 \mathrm{MHz}\right) \delta: 5.68(\mathrm{~s}, 1 \mathrm{H}, \mathrm{CH}), 6.43(\mathrm{~s}, 1 \mathrm{H}$, ArH), 7.18 (d, $J=8.4 \mathrm{~Hz}, 2 \mathrm{H}, \mathrm{ArH}), 7.21 \sim 7.24(\mathrm{~m}, 3 \mathrm{H}$, $\operatorname{ArH}), 7.27$ (d, $J=7.6 \mathrm{~Hz}, 2 \mathrm{H}, \operatorname{ArH}), 7.33$ (d, $J=8.4 \mathrm{~Hz}$, $2 \mathrm{H}, \mathrm{ArH}), 7.47 \sim 7.50(\mathrm{~m}, 1 \mathrm{H}, \mathrm{ArH}), 8.30(\mathrm{~d}, J=8.4 \mathrm{~Hz}$, 1H, ArH), 9.26 (s, 1H, NH), 11.06 (s, 1H, NH), 11.15 (s, $1 \mathrm{H}, \mathrm{NH})$; IR (KBr) v: 3313, 3163, 3105, 3045, 2963, 2930, 2870, 1672, 1639, 1594, 1560, 1488, 1459, 1410, 1350, 1288, 1244, 1102, 1085, 1016, 887, 843, 802, 744, 692 $\mathrm{cm}^{-1}$. HRMS (ESI) calcd for $\mathrm{C}_{24} \mathrm{H}_{17} \mathrm{~N}_{3} \mathrm{OCl}[\mathrm{M}+\mathrm{H}]^{+}$ 398.1062, found 398.1064.

13-(3,5-二甲氧基苯基)-6,13-二氢化-3H-喹啉并 [4,3-b]吡咯并[3,2-f]喹啉-12(11H)-酮(4d): m.p.>300 ${ }^{\circ} \mathrm{C}$; ${ }^{1} \mathrm{H}$ NMR (DMSO- $\left.d_{6}, 400 \mathrm{MHz}\right) \delta: 3.60\left(\mathrm{~s}, 6 \mathrm{H}, 2 \mathrm{OCH}_{3}\right)$, $5.65(\mathrm{~s}, 1 \mathrm{H}, \mathrm{CH}), 6.19(\mathrm{~s}, 1 \mathrm{H}, \mathrm{ArH}), 6.46 \sim 6.49(\mathrm{~m}, 3 \mathrm{H}$, ArH), $7.21 \sim 7.29(\mathrm{~m}, 5 \mathrm{H}, \mathrm{ArH}), 7.46 \sim 7.50(\mathrm{~m}, 1 \mathrm{H}, \mathrm{ArH})$, 8.28 (d, $J=8.0 \mathrm{~Hz}, 1 \mathrm{H}, \operatorname{ArH}), 9.21$ (s, 1H, NH), 11.03 (s, $1 \mathrm{H}, \mathrm{NH}), 11.13$ (s, 1H, NH); IR (KBr) v: 3331, 3223, 2957, 2932, 2887, 2835, 1663, 1591, 1557, 1489, 1465, 1409, 1351, 1309, 1243, 1195, 1094, 1066, 1048, 942, 879, $854,821,783,755,724,692 \mathrm{~cm}^{-1}$. HRMS (ESI) calcd for $\mathrm{C}_{26} \mathrm{H}_{22} \mathrm{~N}_{3} \mathrm{O}_{3}[\mathrm{M}+\mathrm{H}]^{+} 424.1663$, found 424.1670.

13-(3,4-二甲基苯基)-6,13-二氢化-3 $H$-喹啉并 [4,3- $b$ ] 吡咯并[3,2-f]喹啉-12(11H)-酮(4e): m.p. $>300{ }^{\circ} \mathrm{C} ;{ }^{1} \mathrm{H}$ NMR (DMSO- $\left.d_{6}, 400 \mathrm{MHz}\right) \delta: 2.04(\mathrm{~d}, J=6.4 \mathrm{~Hz}, 6 \mathrm{H}$, $\left.2 \mathrm{CH}_{3}\right), 5.61$ (s, 1H, CH), $6.44(\mathrm{~s}, 1 \mathrm{H}, \mathrm{ArH}), 6.86$ (d, $J=8.0$ $\mathrm{Hz}, 1 \mathrm{H}, \mathrm{ArH}), 7.02$ (d, J=8.0 Hz, 1H, ArH), 7.09 (s, 1H, ArH), $7.20 \sim 7.24$ (m, 4H, ArH), 7.27 (d, J=8.0 Hz, 1H, $\operatorname{ArH}), 7.47$ (t, $J=7.6 \mathrm{~Hz}, 1 \mathrm{H}, \operatorname{ArH}), 8.29$ (d, $J=8.0 \mathrm{~Hz}$, 1H, ArH), 9.17 (s, 1H, NH), 11.00 (s, 1H, NH), 11.08 (s, 1H, NH); IR (KBr) v: 3409, 3297, 3174, 3108, 3013, 2963,
2918, 1633, 1613, 1560, 1543, 1501, 1480, 1455, 1390, $1355,1258,1234,1150,1101,812,782,746,722 \mathrm{~cm}^{-1}$. HRMS (ESI) calcd for $\mathrm{C}_{26} \mathrm{H}_{20} \mathrm{~N}_{3} \mathrm{O}[\mathrm{M}-\mathrm{H}]^{-} 390.1606$, found 390.1625 .

13-(2-甲氧基苯基)-6,13-二氢化-3H-喹啉并 [4,3- $b$ ] 吡咯并[3,2-f]喹啉-12(11H)-酮(4f): m.p. 252 254 ${ }^{\circ} \mathrm{C} ;{ }^{1} \mathrm{H}$ NMR (DMSO- $\left.d_{6}, 400 \mathrm{MHz}\right) \delta: 3.90\left(\mathrm{~s}, 3 \mathrm{H}, \mathrm{OCH}_{3}\right), 6.05$ (s, 1H, CH), 6.58 (s, 1H, ArH), $6.63 \sim 6.37$ (m, 1H, ArH), $6.86(\mathrm{~d}, J=8.0 \mathrm{~Hz}, 1 \mathrm{H}, \operatorname{ArH}), 6.94 \sim 6.98(\mathrm{~m}, 1 \mathrm{H}, \operatorname{ArH})$, $7.14 \sim 7.21(\mathrm{~m}, 5 \mathrm{H}, \mathrm{ArH}), 7.24(\mathrm{~d}, J=8.8 \mathrm{~Hz}, 1 \mathrm{H}, \mathrm{ArH})$, $7.43 \sim 7.47$ (m, 1H, ArH), 8.29 (d, $J=8.0 \mathrm{~Hz}, 1 \mathrm{H}, \mathrm{ArH})$, 9.08 (s, 1H, NH), 10.92 (s, 2H, 2NH); IR (KBr) v: 3289, 3094, 2991, 2942, 2899, 2869, 2829, 1661, 1636, 1595, $1560,1490,1461,1421,1352,1332,1290,1242,1157$, 1102, 1031, 865, 799, 751, $696 \mathrm{~cm}^{-1}$. HRMS (ESI) calcd for $\mathrm{C}_{25} \mathrm{H}_{20} \mathrm{~N}_{3} \mathrm{O}_{2}[\mathrm{M}+\mathrm{H}]^{+}$394.1557, found 394.1579.

13-(3-甲氧基苯基)-6,13-二氢化-3H-喹啉并 [4,3- $b$ ] 吡咯并 [3,2-f] 喹啉-12(11H)-酮 (4g): m.p. > $300{ }^{\circ} \mathrm{C} ;{ }^{1} \mathrm{H}$ NMR (DMSO- $\left.d_{6}, 400 \mathrm{MHz}\right) \delta: 3.60\left(\mathrm{~s}, 3 \mathrm{H}, \mathrm{OCH}_{3}\right), 5.65$ (s, 1H, CH), 6.45 (s, 1H, ArH), 6.57 (d, J=8.4 Hz, 1H, $\mathrm{ArH}), 6.84 \sim 6.87(\mathrm{~m}, 2 \mathrm{H}, \mathrm{ArH}), 6.99 \sim 7.02(\mathrm{~m}, 1 \mathrm{H}, \mathrm{ArH})$, $7.22 \sim 7.26(\mathrm{~m}, 5 \mathrm{H}, \mathrm{ArH}), 7.44 \sim 7.46(\mathrm{~m}, 1 \mathrm{H}, \mathrm{ArH}), 8.27$ (d, $J=8.0 \mathrm{~Hz}, 1 \mathrm{H}, \mathrm{ArH}), 9.19$ (s, 1H, NH), 11.00 (s, 1H, $\mathrm{NH}), 11.10(\mathrm{~s}, 1 \mathrm{H}, \mathrm{NH})$; IR (KBr) v: 3346, 3238, 3101, 3053, 2993, 2954, 2870, 2834, 1666, 1605, 1563, 1547, $1488,1460,1397,1355,1264,1240,1132,1099,1052$, $852,811,756,695 \mathrm{~cm}^{-1}$. HRMS (ESI) calcd for $\mathrm{C}_{25} \mathrm{H}_{20} \mathrm{~N}_{3} \mathrm{O}_{2}[\mathrm{M}+\mathrm{H}]^{+}$394.1557, found 394.1572.

13-(4-甲基苯基)-6,13-二氢化-3H-喹啉并 [4,3- $b$ ] 吡 咯并[3,2-f]喹啉-12(11H)-酮(4h): m.p. $>300{ }^{\circ} \mathrm{C} ;{ }^{1} \mathrm{H}$ NMR $\left(\mathrm{DMSO}-d_{6}, 400 \mathrm{MHz}\right) \delta: 2.12\left(\mathrm{~s}, 3 \mathrm{H}, \mathrm{CH}_{3}\right), 5.64(\mathrm{~s}, 1 \mathrm{H}$, $\mathrm{CH}), 6.43$ (s, 1H, ArH), 6.91 (d, $J=8.4 \mathrm{~Hz}, 2 \mathrm{H}, \mathrm{ArH}), 7.19$ (s, 1H, ArH), $7.21 \sim 7.24(\mathrm{~m}, 5 \mathrm{H}, \operatorname{ArH}), 7.27$ (d, $J=7.2$ $\mathrm{Hz}, 1 \mathrm{H}, \mathrm{ArH}), 7.47$ (t, $J=7.6 \mathrm{~Hz}, 1 \mathrm{H}, \mathrm{ArH}), 8.29$ (d, $J=$ $8.4 \mathrm{~Hz}, 1 \mathrm{H}, \mathrm{ArH}), 9.17$ (s, 1H, NH), 11.00 (s, 1H, NH), 11.09 (s, 1H, NH); IR (KBr) v: 3286, 3180, 3016, 2971, 2928, 2896, 2866, 1670, 1603, 1561, 1500, 1487, 1458, 1406, 1349, 1288, 1244, 1161, 1101, 1081, 1047, 886, 852, 803, 780, 746, $723 \mathrm{~cm}^{-1}$. HRMS (ESI) calcd for $\mathrm{C}_{25} \mathrm{H}_{20} \mathrm{~N}_{3} \mathrm{O}[\mathrm{M}+\mathrm{H}]^{+}$378.1608, found 378.1614.

13-(2,4,5-三甲氧基苯基)-6,13-二氢化-3H-喹啉并 [4,3-b]吡咯并[3,2-f]喹啉-12(11H)-酮(4i): m.p.>300 ${ }^{\circ} \mathrm{C}$; ${ }^{1} \mathrm{H}$ NMR (DMSO- $\left.d_{6}, 400 \mathrm{MHz}\right) \delta: 3.43\left(\mathrm{~s}, 3 \mathrm{H}, \mathrm{OCH}_{3}\right)$, $3.67\left(\mathrm{~s}, 3 \mathrm{H}, \mathrm{OCH}_{3}\right), 3.89\left(\mathrm{~s}, 3 \mathrm{H}, \mathrm{OCH}_{3}\right), 5.95(\mathrm{~s}, 1 \mathrm{H}, \mathrm{CH})$, $6.56(\mathrm{~s}, 1 \mathrm{H}, \mathrm{ArH}), 6.60 \sim 6.61(\mathrm{~m}, 1 \mathrm{H}, \mathrm{ArH}), 6.75(\mathrm{~s}, 1 \mathrm{H}$, 
ArH), $7.17 \sim 7.21(\mathrm{~m}, 3 \mathrm{H}, \operatorname{ArH}), 7.23$ (d, $J=8.0 \mathrm{~Hz}, 1 \mathrm{H}$, ArH), 7.27 (d, $J=8.4 \mathrm{~Hz}, 1 \mathrm{H}, \operatorname{ArH}), 7.45 \sim 7.49$ (m, 1H, ArH), 8.30 (d, J=8.4 Hz, 1H, ArH), 9.13 (s, 1H, NH), 10.93 (s, 1H, NH), 10.96 (s, 1H, NH); IR (KBr) v: 3364, 3286, 3100, 2950, 2937, 2871, 2833, 1669, 1638, 1597, $1550,1506,1462,1429$, 1390, 1352, 1315, 1243, 1206, $1098,1032,865,798,753 \mathrm{~cm}^{-1}$. HRMS (ESI) calcd for $\mathrm{C}_{27} \mathrm{H}_{24} \mathrm{~N}_{3} \mathrm{O}_{4}[\mathrm{M}+\mathrm{H}]^{+}$454.1768, found 454.1787.

\section{3 产物 $4 \mathrm{~b}$ 单晶的实验方法}

晶体学数据和衍射强度数据的收集在 Bruker 型四 圆衍射仪上进行. 将 $0.176 \mathrm{~mm} \times 0.142 \mathrm{~mm} \times 0.098 \mathrm{~mm}$ 的无色长方形单晶置于装有单色器的四圆衍射仪上, 用 Mo K $\alpha(\lambda=0.71073 \AA)$ 幅射和 $\varphi-\omega$ 扫描方式收集衍射数 据，在 $5.90^{\circ}<2 \theta<50.04^{\circ}$ 范围内共收集 4414 个独立衍 射点, 可观测衍射点 3302 个 $[I>2 \sigma(I)]$, 数据经 Lp 校正. 晶体结构由直接法 (SHELXTL 程序) 解出, 经多轮 Fourier 合成获得全部非氢原子, 全部氢原子由理论后 直接加氢而得到. 全部非氢原子的坐标及各向异性热参 数, 采用 $w=1 /\left[\sigma^{2}\left(F_{\mathrm{o}}{ }^{2}\right)+(0.0778 P)^{2}+3.2673 P\right], P=$ $\left(F_{\mathrm{o}}{ }^{2}+2{F_{\mathrm{C}}}^{2}\right) / 3$, 经全矩阵最小二乘法修正及收玫. 最终 的偏离因子为 $R=0.0495, w R=0.0672$. 最终差值电子密 度的最高峰为 $0.460 \mathrm{e} \cdot \AA^{-3}$, 最低峰为 $-0.471 \mathrm{e} \cdot \AA^{-3}$.

$(\Delta / \sigma)_{\max }=0.000, S=1.032$.

辅助材料(Supporting Information) 化合物 $\mathbf{4 a} \sim \mathbf{4 i}$ 的 ${ }^{1} \mathrm{H}$ NMR 图谱和化合物 $\mathbf{4 b}$ 的 cif 文件. 这些材料可以免 费从本刊网站(http://sioc-journal.cn)上下载.

\section{References}

[1] (a) Keri, R. S.; Patil, S. A. Biomed. Pharmacother. 2014, 68, 1161. (b) Muregi, F. W.; Wamakima, H. N.; Kimani, F. T. Curr. Pharm. Des. 2012, 18, 3505.

(c) Chauhan, S. S.; Sharma, M.; Chauhan, P. M. S. Drug News Perspect 2010, 23, 632.

(d) Weissbuch, I.; Leiserowitz, L. Chem. Rev. 2008, 108, 4899.

[2] Pascolo, S. Eur. J. Pharm. 2016, 771, 139.

[3] Babalonis, S.; Hampson, A. J.; Lofwall, M. R.; Nuzzo, P. A.; Walsh,
S. L. J. Clin. Pharm. 2015, 55, 1332.

(a) Zhang, S.-S.; Jiang, C.-Y.; Wu, J.-Q.; Liu, X.-G.; Li, Q.; Huang, Z.-S.; Li, D.; Wang, H. Chem. Commun. 2015, 51, 10240.

(b) Tan, C.; Xiang, H.; He, Q.; Yang, C. Eur. J. Org. Chem. 2015, (17), 3656

(c) Heravi, M. M.; Hosseini, M.; Oskooie, H. A.; Baghernejad, B.; Farzaneh, F. Chin. J. Chem. 2010, 28, 2045.

(d) Ranjbar-Karimi, R.; Hashemi-Uderji, S.; Bazmandegan-Shamili, A. Chin. J. Chem. 2011, 29, 1624.

(e) Du, B. X.; Li, Y. L.; Wang, X. S.; Zhang, M. M.; Shi, D. Q.; Tu, S. J. Chin. J. Org. Chem. 2006, 26, 698 (in Chinese).

(杜百祥, 李玉玲, 王香善, 张梅梅, 史达清, 屠树江, 有机化学, 2006, 26, 698.)

(f) Li, Y. X. Chin. J. Org. Chem. 2013, 33, 2334 (in Chinese).

(李元祥, 有机化学, 2013, 33, 2334.)

[4] (a) Chen, Y.; Tu, S. J.; Jiang, B.; Li, C. M. Chin. J. Org. Chem. 2007, 27, 1288 (in Chinese).

(陈艳，屠树江，姜波，李春梅，有机化学, 2007, 27, 1288.)

(b) Hua, G. P.; Xu, J. N.; Tu, S. J.; Wang, Q.; Zhang, J. P.; Zhu, X. T.; Li, T. J.; Zhu, S. L.; Zhang, X. J. Chin. J. Org. Chem. 2005, 25, 1610 (in Chinese).

(华国平，徐佳宁，屠树江,王倩，张金鹏，朱晓肜，李团结，朱松 磊, 章晓镜, 有机化学, 2005, 25, 1610.)

(c) Liu, J.; Liu, G.; Tian, X. H.; Cao, L. H. Chin. J. Org. Chem. 2008, 28, 73 (in Chinese).

(刘俊, 刘罡, 田晓红, 曹玲华, 有机化学, 2008, 28, 73.)

(d) Shi, D. Q.; Shi, J. W.; Yao, H.; Jiang, H.; Wang, X. S. Chin. J. Org. Chem. 2008, 28, 261 (in Chinese).

(史达清, 拾景文, 姚浩, 蒋虹, 王香善, 有机化学, 2008, 28, 261.)

[5] (a) Wang, X. S.; Zhang, M. M.; Zeng, Z. S.; Shi, D. Q.; Tu, S. J.; Wei, X. Y.; Zong, Z. M. Tetrahedron Lett. 2005, 46, 7169.

(b) Peng, J.; Hao, W.; Wang, X.; Tu, S. J.; Ma, N.; Zhang, G. Chin. J. Chem. 2009, 27, 1707.

(c) Zhou, Y. J.; Chen, D. S.; Li, Y. L.; Liu, Y.; Wang, X. S. ACS Comb. Sci. 2013, 15, 498.

(d) Shi, F.; Yan, S.; Zhou, D.; Tu, S.; Zou, X.; Hao, W.; Zhang, X.; Han, Z.; Wu, S.; Cao, X. J. Heterocycl. Chem. 2009, 46, 563.

[6] (a) Chen, D. S.; Zhou, Y. J.; Li, Y. L.; Yao, C. S.; Wang, X. S. Comb. Chem. High Throughput Screening 2013, 16, 550.

(b) Li, C.; Mu, X. Y.; Li, Y. L. Liu, Y.; Wang, X. S. ACS Comb. Sci. 2013, 15, 267.

(c) Zhang, M. M.; Wang, W.; Wang, X. S. J. Heterocycl. Chem. 2014, 51, E349.

(d) Lu, W. Q.; Zhuang, R.; Chen, D. S.; Wang, X. S. Polycyclic Aromat. Compd. 2014, 34, 606. 